\title{
Combined non-invasive imaging for predicting cardiovascular events
}

\section{Is three a crowd?}

\author{
Austin A. Robinson, MD, , a,b and Jamieson M. Bourque, MD, MHS, FASNC ${ }^{\mathrm{a}, \mathrm{b}}$ \\ a Cardiovascular Division, Department of Medicine, University of Virginia Health System, \\ Charlottesville, VA \\ b Cardiovascular Imaging Center, University of Virginia Health System, Charlottesville, VA
}

Received Sep 30, 2016; accepted Sep 30, 2016

doi:10.1007/s12350-016-0719-5

\section{See related article, pp. 833-841}

In this issue of the Journal, Nappi et al analyze the incremental prognostic value of coronary artery calcium scoring (CACS), single-photon emission computed tomography myocardial perfusion imaging (SPECT MPI), and coronary CT angiography (CCTA) in a prospective cohort of patients with low-to-intermediate probability of coronary artery disease (CAD) who were referred clinically for all three studies. The authors excluded those who were revascularized within 2 months, setting aside the question of diagnostic accuracy. This is the first study to prospectively and contemporaneously perform all three modalities and compare their prognostic value. The authors are to be applauded for setting out to accomplish such an ambitious goal and for obtaining near-complete follow-up.

Despite low numbers of patients and events, the authors were able to demonstrate incremental prognostic value with SPECT over CACS. But in whom? The population studied by Nappi et al consisted of patients deemed at low or intermediate pre-test probability for CAD according to extended Diamond-Forrester classification. The percentage breakdown between low and intermediate groups is not available. Interestingly, the majority of patients $(85 / 156,54.5 \%)$ were asymptomatic prior to testing. The population studied stands in contrast

Reprint requests: Jamieson M. Bourque, MD, MHS, FASNC, Cardiovascular Division, Department of Medicine, University of Virginia Health System, 1215 Lee Street, Box 800158, Charlottesville, VA 22908; jamieson2@gmail.com

J Nucl Cardiol 2018;25:842-4.

$1071-3581 / \$ 34.00$

Copyright (C) 2016 American Society of Nuclear Cardiology. to those in other trials of non-invasive imaging, such as PROMISE, in which the patients were symptomatic by study design and had a substantial mean combined Diamond-Forrester and Coronary Artery Surgery Study risk score of $53 \pm 21 \%$. ${ }^{1}$ The authors do not address the reasons these asymptomatic individuals underwent imaging. Current appropriate use criteria provide limited situations for testing of asymptomatic individuals. ${ }^{2}$ Thus, the authors' indications for testing, as well as the relative order of testing modalities, could provide insight on the role of non-invasive imaging in this patient population.

In the patient population studied, this analysis offers several important results. Using a composite outcome of cardiac death, non-fatal myocardial infarction and unstable angina requiring revascularization, the authors noted that CACS, SPECT MPI, and CCTA all demonstrated prognostic value independently. However, CCTA did not provide incremental prognostic information after CACS and SPECT findings were combined with clinical data. The chi-square value of clinical data in the incremental analysis seems low. It is unlikely that exercise capacity, one of the most powerful prognostic markers in exercise MPI, was included. ${ }^{3}$ Patients able to achieve a high exercise workload of $\geq 10$-METS have an excellent prognosis, and inclusion of this variable in the clinical model may have altered the incremental value of all three imaging modalities. ${ }^{4}$ However, despite potential limitations, the results highlight a potential role for SPECT MPI. There is an emerging literature on the prognostic value of CACS in patients with normal SPECT, but this report provides a novel demonstration of the additive prognostic value of SPECT over $\mathrm{CACS}^{5-7}$ and lends further credibility to the idea that ischemia is an important prognostic marker. ${ }^{8}$ 
In this predominately asymptomatic, low-to-intermediate risk cohort, the data make a compelling case for the utility of CACS as an initial modality for risk evaluation in ischemic heart disease; there were no events among patients with a CACS of 0 . This finding provides additional confirmation of the excellent prognosis of patients with a negative calcium score. ${ }^{9,10}$ In such patients, there appears to be little role for SPECT MPI or CCTA. In those with CACS $>0$, there may be incremental value in functional imaging with SPECT MPI. However, the present results suggest that CCTA may find itself the third wheel in prognostication. On the other hand, the finding of a significant reduction in event-free survival in patients with matched defects (MPI defects in a coronary territory supplied by a vessel with CCTA stenosis) vs unmatched defects suggests there is likely a role for hybrid imaging in some patients. ${ }^{11,12}$

This study has other limitations acknowledged by the authors. It was performed in a single center and included a relatively small number of patients. Furthermore, the combined endpoint was driven largely by unstable angina requiring revascularization, accounting for $73 \%$ of all events. There were relatively few cardiac deaths or non-fatal myocardial infarctions. The percentage of "hard" outcomes is lower than in other studies of MPI and prognosis. ${ }^{13}$ While unstable angina requiring revascularization is an important endpoint, the makeup of events further emphasizes the lower risk of this cohort. The low number of events did not prevent SPECT MPI from having incremental value. However, a higher event rate may have resulted in incremental benefit to CCTA, especially given the significant Kaplan-Meier analysis with matched defects and the trend towards significance in the incremental Chi-square analysis $(P=0.075)$.

An important additional finding was that nearly all events (20 of $22,91 \%$ ) occurred in men despite women making up $37 \%$ of the population. The clustering of events in men further underscores the difficult task of sex-specific prognostication. In patients with abnormal SPECT MPI, a recent study observed higher rates of cardiac death and non-fatal MI as well as coronary revascularization among men, compared with women. ${ }^{14}$ Data on CACS have been disparate. An older metaanalysis of 20,178 patients in 3 large studies showed similar CACS-based mortality risk between women and men. ${ }^{15}$ However, a more recent study that included 15year mortality rates suggested increased risk among women with higher CACS. ${ }^{16}$ Multi-modal evaluation further complicates this issue and, unfortunately, the findings presented by Nappi et al may not be fully applicable to women.

Many questions persist. It remains to be seen whether the benefit of SPECT MPI over CACS and lack of value from added CCTA analysis will be found in patients with higher pre-test probability of CAD, or among groups with a greater symptom burden. Factoring in exercise capacity, is there a prognostic role for imaging in patients with good functional capacity $(\geq 10$ METs)? When should hybrid imaging be performed? Are these results applicable to women? These important questions await the efforts of future investigators to help define optimal risk management in patients at risk for ischemic heart disease.

\section{Disclosure}

Jamieson M. Bourque receives research grant support from Astellas Pharma. Austin A. Robinson has no disclosures to report.

\section{References}

1. Douglas PS, Hoffmann U, Patel MR, Mark DB, Al-Khalidi HR, Cavanaugh $\mathrm{B}$, et al. Outcomes of anatomical versus functional testing for coronary artery disease. N Engl J Med. 2015;372:1291300 .

2. Wolk MJ, Bailey SR, Doherty JU, Douglas PS, Hendel RC, Kramer CM, et al. ACCF/AHA/ASE/ASNC/HFSA/HRS/SCAI/ SCCT/SCMR/STS 2013 multimodality appropriate use criteria for the detection and risk assessment of stable ischemic heart disease: A report of the American College of Cardiology Foundation Appropriate Use Criteria Task Force, American Heart Association, American Society of Echocardiography, American Society of Nuclear Cardiology, Heart Failure Society of America, Heart Rhythm Society, Society for Cardiovascular Angiography and Interventions, Society of Cardiovascular Computed Tomography, Society for Cardiovascular Magnetic Resonance, and Society of Thoracic Surgeons. J Am Coll Cardiol. 2014;63:380-406.

3. Bourque JM, Beller GA. Value of exercise ecg for risk stratification in suspected or known CAD in the era of advanced imaging technologies. JACC Cardiovasc Imaging. 2015;8:1309-21.

4. Bourque JM, Charlton GT, Holland BH, Belyea CM, Watson DD, Beller GA. Prognosis in patients achieving $\geq 10$ METS on exercise stress testing: Was SPECT imaging useful? J Nucl Cardiol. 2011;18:230-7.

5. Schenker MP, Dorbala S, Hong ECT, Rybicki FJ, Hachamovitch $\mathrm{R}$, Kwong RY, et al. Interrelation of coronary calcification, myocardial ischemia, and outcomes in patients with intermediate likelihood of coronary artery disease. Circulation. 2008;117:1693700 .

6. Chang SM, Nabi F, Xu J, Peterson LE, Achari A, Pratt CM, et al. The coronary artery calcium score and stress myocardial perfusion imaging provide independent and complementary prediction of cardiac risk. J Am Coll Cardiol. 2009;54:1872-82.

7. Engbers EM, Timmer JR, Ottervanger JP, Mouden M, Knollema S, Jager PL. Prognostic value of coronary artery calcium scoring in addition to single-photon emission computed tomographic myocardial perfusion imaging in symptomatic patients. Circ Cardiovasc Imaging 2016 (Epub ahead of print).

8. Shaw LJ, Iskandrian AE. Prognostic value of gated myocardial perfusion SPECT. J Nucl Cardiol. 2004;11:171-85.

9. Arad Y, Goodman KJ, Roth M, Newstein D, Guerci AD. Coronary calcification, coronary disease risk factors, $\mathrm{C}$-reactive protein, and 
atherosclerotic cardiovascular disease events: The St. Francis Heart Study. J Am Coll Cardiol. 2005;46:158-65.

10. Valenti V, O'Hartaigh B, Heo R, Cho I, Schulman-Marcus J, Gransar H, et al. A 15-year warranty period for asymptomatic individuals without coronary artery calcium: A prospective followup of 9,715 individuals. JACC Cardiovasc Imaging. 2015;8:900-9.

11. Kaufmann PA, Di Carli MF. Hybrid SPECT/CT and PET/CT imaging: The next step in noninvasive cardiac imaging. Semin Nucl Med. 2009;39:341-7.

12. Gaemperli O, Bengel FM, Kaufmann PA. Cardiac hybrid imaging. Eur Heart J. 2011;32:2100-8.

13. Yoshinaga K, Chow BJW, Williams K, Chen L, Garrard L, Szeto $\mathrm{AL}$, et al. What is the prognostic value of myocardial perfusion imaging using rubidium-82 positron emission tomography? J Am Coll Cardiol. 2006;48:1029-39.
14. Romero-Farina G, Candell-Riera J, Ferreira-González I, AguadéBruix S, Pizzi N, García-Dorado D. Normal myocardial perfusion gated SPECT and positive stress test: Different prognoses in women and men. J Nucl Cardiol. 2015;22:453-65.

15. Bellasi A, Lacey C, Taylor AJ, Raggi P, Wilson PW, Budoff MJ, et al. Comparison of prognostic usefulness of coronary artery calcium in men versus women (results from a meta- and pooled analysis estimating all-cause mortality and coronary heart disease death or myocardial infarction). Am J Cardiol. 2007;100:409-14.

16. Kelkar AA, Schultz WM, Khosa F, Schulman-Marcus J, O'Hartaigh BW, Gransar $\mathrm{H}$, et al. Long-term prognosis after coronary artery calcium scoring among low-intermediate risk women and men. Circ Cardiovasc Imaging 2016 (Epub ahead of print). 